\title{
Foot-and-mouth disease virus serotypes detected in Tanzania from 2003 to 2010: Conjectured status and future prospects
}

\author{
Authors: \\ Christopher J. Kasanga ${ }^{1}$ \\ Raphael Sallu \\ Fredrick Kivaria ${ }^{3}$ \\ Mathias Mkama² \\ Joseph Masambu² \\ Mmeta Yongolo² \\ Sachindra Das ${ }^{2}$ \\ Chanasa Mpelumbe-Ngeleja² \\ Philemon N. Wambura \\ Donald P. King ${ }^{4}$ \\ Mark M. Rweyemamu ${ }^{1}$

\section{Affiliations:} \\ ${ }^{1}$ Faculty of Veterinary \\ Medicine, Sokoine University \\ of Agriculture, Tanzania \\ ${ }^{2}$ Central Veterinary \\ Laboratory, Dar-es-Salaam, \\ Tanzania \\ ${ }^{3}$ National Epidemiology \\ Unit, Ministry of Livestock \\ Development and Fisheries, \\ Dar-es-Salaam, Tanzania \\ ${ }^{4}$ Institute for Animal Health, \\ Woking, United Kingdom

\section{Correspondence to:} \\ Christopher Kasanga \\ Email: \\ christopher.kasanga@sacids. \\ org

\section{Postal address:} \\ PO Box 3019, Sokoine \\ University of Agriculture, \\ Morogoro \\ How to cite this proceeding \\ Kasanga, C.J., Sallu, R., \\ Kivaria, F., Mkama, M. \\ Masambu, J., Yongolo, M. et \\ al., 'Foot-and-mouth disease \\ virus serotypes detected in \\ Tanzania from 2003 to 2010: \\ Conjectured status and future \\ prospects', Onderstepoort \\ Journal of Veterinary Research \\ 79(2), Art. \#462, 4 pages. \\ http://dx.doi.org/10.4102/ \\ ojvr.v79i2.462 \\ Note: \\ Proceedings of the \\ Conference of the Southern \\ African Centre for Infectious \\ Disease Surveillance 'One \\ Health' held at the National \\ Institute for Communicable \\ Diseases, Johannesburg, July \\ 2011.
}

This study was conducted to investigate the presence of foot-and-mouth disease virus (FMDV) in different geographic locations of Tanzania. Epithelial tissues and fluids $(n=364)$ were collected from cattle exhibiting oral and foot vesicular lesions suggestive of FMD and submitted for routine FMD diagnosis. The analysis of these samples collected during the period of 2002 and 2010 was performed by serotype-specific antigen capture ELISA to determine the presence of FMDV. The results of this study indicated that 167 out of $364(46.1 \%)$ of the samples contained FMDV antigen. Of the 167 positive samples, 37 (28.4\%) were type O, 7 (4.1\%) type A, 45 (21.9\%) SAT 1 and 79 (45.6\%) SAT 2. Two FMDV serotypes (O and SAT 2) were widely distributed throughout Tanzania whilst SAT 1 and A types were only found in the Eastern zone. Our findings suggest that serotypes A, O, SAT 1 and SAT 2 prevail in Tanzania and are associated with the recent FMD outbreaks. The lack of comprehensive animal movement records and inconsistent vaccination programmes make it difficult to determine the exact source of FMD outbreaks or to trace the transmission of the disease over time. Therefore, further collection and analysis of samples from domestic and wild animals are being undertaken to investigate the genetic and antigenic characteristics of the circulating strains, so that a rational method to control FMD in Tanzania and the neighbouring countries can be recommended.

\section{Introduction}

Foot-and-mouth disease virus (FMDV; family Picornaviridae, genus: Aphthovirus) exists as seven serotypes (O, A, C, Asia 1, Southern African Territories 1-3 [SAT 1-3]) and causes a highly contagious disease of ruminants and swine. Foot-and-mouth disease (FMD) is endemic in most of sub-Saharan Africa and is considered to be one of the most widely distributed transboundary animal diseases (TAD) in the world (OIE and FAO Reports 2003). In Tanzania, FMD is the most important viral TAD (Swai et al. 2009). FMDV in endemic settings across the world have been categorised into six pools; each comprising a different geographic location with different predominant serotypes. The FMDV pools include pool 1 in East Asia ( $\mathrm{O}$, A and Asia 1), pool 2 in Central Asia (O, A and Asia 1), pool 3 in Europe and South Asia (O, A and Asia 1), pool 4 in Southern, Eastern and Horn of Africa (A, O, SAT 1, 2 and 3), pool 5 in Western Africa (O, A, SAT 1 and 2), and pool 6 in Southern Africa (SAT 1, 2 and 3) (Paton, Sumption \& Charleston 2009). Tanzania links East Africa and southern Africa in a region that overlaps between pools 4 and 6 .

Since its first documentation in 1927 and first isolation of the virus in 1954, many FMD outbreaks have occurred across different areas of Tanzania. Unrestricted animal movements are an important mechanism by which FMD is spread within and across international borders (Di Nardo, Knowles \& Paton 2011; Kivaria 2003). In order to limit the spread and economic impact of the disease, the control measures implemented during outbreaks in Tanzania typically consist of quarantine and restriction of animal movements particularly in areas with well-defined farming systems (Kivaria 2003). However, the presence of multiple FMD serotypes, and the occurrence of subclinical forms of the disease renders FMD control very difficult, particularly in pastoral agricultural systems where resources are limited. Establishing and quantifying the distribution of FMDV serotypes in different eco-climatic regions in the country will contribute to the understanding of FMD epidemiology, and provide knowledge to researchers, vaccine manufacturers and policy makers to more efficiently deploy resources to control FMD field outbreaks.

Of the seven known FMDV serotypes, four (O, A, SAT 1 and SAT 2) have been previously identified and reported in Tanzania (Mlangwa 1983; Rweyemamu \& Loretu 1972; Rweyemamu et al. 2008b; Swai, Mrosso \& Masambu 2009; Vosloo et al. 2002). Despite the fact that FMD is endemic in Tanzania, only limited studies have been conducted to describe the spatial and temporal

Copyright: (C) 2012. The Authors. Licensee: AOSIS OpenJournals. This work is licensed under the Creative Commons Attribution License. 
distribution of FMD outbreaks (Picado et al. 2010) and FMDV serotypes in the country (Rweyemamu \& Loretu 1972; Swai et al. 2009). Furthermore, no specific detailed studies have been undertaken to describe the molecular epidemiology of Tanzanian FMDV serotypes. These approaches can define the distribution patterns of viruses and factors determining endemicity of FMD in Tanzania, and data from such studies will provide important information that will underpin future efforts to control FMD. Therefore, the aim of this current study was to use routine laboratory diagnostic methods to establish the spatial and temporal distribution of FMDV serotypes in different regions of Tanzania. Sample collection targeted FMD outbreaks that occurred in the country between 2003 and 2010 .

\section{Materials and methods}

\section{Sample collection}

All samples were collected from confirmed FMD outbreaks (based on clinical signs) in cattle in different regions of Tanzania. The samples were collected by staff from seven strategically placed veterinary investigation centres in the country. These samples were obtained from regions namely Arusha and Kilimanjaro (Northern zone), Mwanza and Kagera (Northern-Lake zone), Tabora, Rukwa and Kigoma (Western zone), Mtwara (Southern-coastal zone), Iringa and Mbeya (Southern zone), Dodoma and Singida (Central zone) and Dar-es-Salaam, Pwani and Morogoro (Eastern-Coastal zone). Epithelial tissues and fluid from oral and foot lesions were collected and immediately placed in virus transport media composed of equal amounts of sterile glycerol $(50 \%$ $\mathrm{v} / \mathrm{v}$ ) and $0.04 \mathrm{M}$ phosphate buffered saline at $\mathrm{pH} 7.2-\mathrm{pH} 7.6$, stored at $+4{ }^{\circ} \mathrm{C}$ and transported to the Central Veterinary Laboratory (CVL), Dar-es-Salaam.

\section{Laboratory analysis of samples}

Vesicular fluids and tissue epithelia were analysed for FMDV antigens, and the viral antigen typed into different serotypes by the antigen detection ELISA (IAH, Pirbright) as previously described (Hamblin, Armstrong \& Hedger 1984; Ferris \& Dawson 1988).

\section{Data analysis}

The dependent variable tested was the seropositivity to FMDV antigen (positive or negative) and specific serotype, in this case types A, O, SAT 1 and SAT 2 . The independent variables investigated were the geographic location of the origin of samples and/or FMD outbreaks.

\section{Results}

A total of 364 samples from different eco-climatic regions in Tanzania were examined for FMDV antigen. Of the tested samples, 167 (45.9\%) were positive to at least one of the four serotypes of FMDV. Of the 167 positive samples, 37 (22.0\%) were serotype O, $7(4.2 \%)$ serotype A, 45 (27\%) serotype SAT 1 and 78 (46.8\%) serotype SAT 2 (Table 1). There were no samples that tested positive for more than one FMDV serotype. Serotype SAT 2 was identified throughout the Northern, Southern, Western, Eastern and Central zones of Tanzania (Figure 1). Serotypes O, SAT 1 and SAT 2 were identified at least from one geographic region every year from 2003 to 2010 (Table 1). Serotype A was exclusively found in the Eastern coastal zone (Figure 1), and was only detected more recently in 2009 (Table 1). Interestingly, serotype SAT 1 was consistently detected only in the Eastern coastal region throughout the eight years (2003-2010). Serotype O was mainly found in the Southern and Northern highlands as well as the Lake zones. Animals from which all the tested samples were obtained had no documented history or evidence of vaccination against FMDV.

\section{Discussion}

FMD is known to be endemic in Tanzania. These findings show that FMDV is widely distributed in many parts of the country with at least four serotypes being found. The four serotypes that were detected during the period of from 2003 to 2010 were O, A, SAT 1 and SAT 2. This is broadly

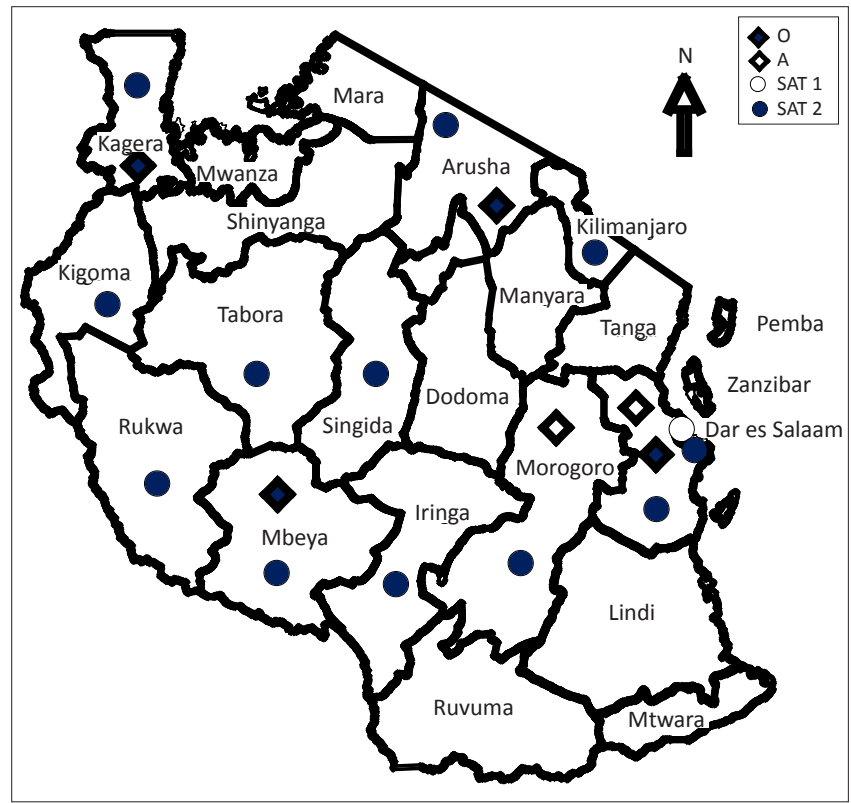

FIGURE 1: Geographic distribution of foot-and-mouth disease virus serotypes detected in Tanzania between 2003 and 2010.

TABLE 1: Summary of foot-and-mouth disease virus serotypes detected in Tanzania from 2003 to 2010.

\begin{tabular}{lllllcc}
\hline Year & $\begin{array}{l}\text { Samples } \\
\text { tested }\end{array}$ & $\begin{array}{l}\text { Samples } \\
\text { positive } \dagger\end{array}$ & \multicolumn{6}{c}{ Serotypes } \\
\cline { 5 - 7 } & & & O & A & SAT 1 & SAT 2 \\
\hline 2003 & 42 & 19 & 2 & 0 & 5 & 12 \\
2004 & 39 & 19 & 4 & 0 & 6 & 9 \\
2005 & 28 & 10 & 1 & 0 & 3 & 6 \\
2006 & 57 & 27 & 10 & 0 & 4 & 13 \\
2007 & 45 & 29 & 12 & 0 & 7 & 10 \\
2008 & 35 & 15 & 4 & 0 & 4 & 7 \\
2009 & 60 & 27 & 2 & 7 & 6 & 13 \\
\hline 2010 & 58 & 21 & 2 & 0 & 10 & 9 \\
\hline Total & 364 & - & 37 & 7 & 45 & 78 \\
\hline
\end{tabular}

$\dagger$, Samples that were positive to any of the four detected serotypes; A, O, SAT1 and SAT2. 
in agreement with findings from previous studies, which reported the detection of these serotypes in this country from late 1950s (Rweyemamu \& Loretu 1972; Vosloo et al. 2002). These observations suggest that serotypes O, A, SAT 1 and SAT 2 have been, and are also associated with the recent FMD outbreaks in different areas of Tanzania.

We have observed that the three serotypes (O, SAT 1 and SAT 2) were detected consistently every year from 2003 to 2010 (Table 1). Studies conducted by others have reported the existence of serotypes $\mathrm{O}$ and SAT 2 in Tanzania to be as far back as in 1950s with the detection of SAT 1 for the first time in 1971 (Rweyemamu \& Loretu 1972, 1973). Together, these findings indicate that types $\mathrm{O}$ and SAT 2 are old in Tanzania and have consistently been observed in the country. These observations raise some questions such as, what factors could be involved in the successive existence of these serotypes? Are the viruses detected in 1950s genetically and antigenically related to the most recent strains? Are viruses from different geographic regions genetically related? All these call for a need of performing a detailed study on the molecular epidemiology of FMDV in Tanzania.

The current and previous studies indicate some enigma relating to the dynamics of Serotype A in Tanzania. During the period covered by this study (i.e. from 2003 to 2010) it was detected only in 2009 in the Eastern coastal zone. Rweyemamu and Loretu (1972) reported regular detection of this sero-type in the Northern, Northern-lake and Central zones of Tanzania for up to 1971. Swai et al. (2009), however, did not find serotype $\mathrm{A}$ in the samples collected between 1997 and 2004. The factors that may have led to the apparent disappearance of serotype A in Tanzania for possibly up to 38 years (from 1971 up to 2009) have not been elucidated. They could include high fragility of the virus and low number of samples that were brought for analysis. However, the reappearance of the type A viruses could possibly be a consequence of cross-border spread of the virus from the neighbouring countries, implying that unlike serotypes $\mathrm{O}$, SAT 1 and SAT 2, serotype A might not be truly endemic in Tanzania. Similar observations have also been made in Iran that reported the emergence of serotype A sub-lineage 'A-Iran-05' (after long time of no periodic outbreaks of type A viruses), which has no closely related antecedents (Knowles et al. 2009). Together, these observations indicate the possibility of emergence of different serotype A strains in different geographic areas. Therefore further studies are required to determine the genetic characteristics of the old and new serotype A isolates detected in Tanzania. This will unravel the evolutionary characteristics of type A sublineages, which could be useful for the control of emerging type A variants in the region.

As the government of the United Republic of Tanzania is planning to adopt a strategic control programme for FMD through vaccination and controlled animal movements, a wider knowledge and understanding of FMDV dynamics and epidemiology should be taken into consideration. This will require the identification of high-risk 'hotspots' as well as potentially infected and FMD-free zones. This task will however need considerable political commitment and laboratory resources to define the spatial and temporal distribution of FMD outbreaks and serotypes in the country. The heterogeneity of FMDV serotypes observed in this study (Table 1) and their spatial occurrence (Figure 1) highlights the need for continuous surveillance of FMD so as to monitor the infection status and spread of FMDV serotypes in livestock and wildlife in Tanzania. As risk factors and transmission characteristics differ in each region (Swai et al. 2009; Picado et al. 2010), a regional FMD surveillance system involving Tanzania and the neighbouring countries will provide information on serotype spatiotemporal distribution for effective control of the disease.

Serotype SAT 2 was detected almost throughout the country for the whole period of 2003-2010 (Table 1), with the highest detection rate of $46.8 \%$. Additional information from Veterinary Investigation Centres (VICs) in Tanzania (data not shown) indicates that SAT 2 strains were obtained from regions with relatively higher number of livestock, especially cattle. These findings suggest that SAT 2 could be incriminated as the cause of many FMD outbreaks in Tanzania. Efforts to isolate and characterise the viruses through genome sequencing are required so as to understand the genetic and antigenic features of field strains; information that is necessary for selection of appropriate vaccine candidate strains for use in Tanzania and neighbouring countries.

Serotype O seems to be widespread in the Northern, Southern, Western and Eastern zones, and not in the central parts of Tanzania (Figure 1). This observation agrees with the previous reports in Tanzania (Mlangwa 1983; MoWLD 2003). In addition, serotypes A and SAT 1 were also not detected from the central parts of Tanzania. However, it is important to recognise that the absence of serotypes $\mathrm{O}, \mathrm{A}$ and SAT 1 from the central parts of Tanzania could be due to low number of samples submitted to CVL for diagnosis that may be related to under reporting of outbreaks, inappropriate cold chain facilities and logistics rather than the actual situation of disease occurrence.

The control of FMD, especially following outbreaks, requires timely identification and characterisation of circulating FMDV serotypes in a given geographic area. Tanzania is found in the Great Lakes (also known as the East African Community (EAC) or Southern-East Africa FMD epidemiological cluster characterised by a substantial diversity of circulating strains or topotypes (Rweyemamu et al. 2008a).

Five serotypes (A, O, C, SAT 1 and SAT 2) are known to be endemic in this cluster (Sahle et al. 2007; Vosloo et al. 2002), and a sixth serotype (SAT 3) has only been isolated from African buffaloes in Uganda in 1970 (Hedger, Forman \& Woodford 1973). In the current study, we detected serotypes A, O, SAT 1 and SAT 2 in cattle from different geographic locations in Tanzania. So far serotypes C and SAT 3 have never been detected in Tanzania. Whether these serotypes, that have been reported to prevail in the EAC cluster, also exist in Tanzania remain unclear and need further systematic investigation.

The current spatiotemporal distribution of FMDV serotypes reported in this study indicates the absence of FMDV in 
southeastern areas of Tanzania bordering Mozambique (Figure 1). This area has also been designated as low-density FMD area with no evidence of FMD outbreaks (Picado et al. 2010). These findings suggest that the southeastern corner, precisely the Mtwara corridor, could be considered as a potential FMD-free zone whereby strict surveillance and control programmes should be implemented for production of livestock meant for exportation.

In this study we have observed an inconsistency serotype distribution in Tanzania (Figure 1). The heterogenic distribution of the different serotype could be ascribed to several factors such as presence of diverse susceptible wildlife reservoirs, inadequate diagnostic capacities, diverse farming systems, socio-economic factors, uncontrolled animal movements, genetic and antigenic variation of the pathogen as well as lack of clear disease control policies (Rweyemamu 1984). However, the laboratory capacity in Tanzania and neighbouring countries has been considered to be inadequate in terms of facilities, equipment and diagnostic kits. Therefore, enhancement of laboratory capacity to undertake disease surveillance is of a paramount importance so as to keep pace with the accurate and timely identification of FMDV field strains, which is required for appropriate control strategies of FMD in the region.

We did not test any samples from wildlife in this study as all samples were obtained from FMD outbreaks in cattle. However, the epidemiological role of wildlife such as the African buffalo (Syncerus caffer) as carriers of particularly the SAT serotypes has been widely discussed (Hedger et al. 1973; Vosloo et al. 1996). A number of regions from which samples were derived are known to be potentially livestockwildlife interface areas with predominantly high population of buffalos. Further studies are required to elucidate the epidemiological link of FMDV maintenance in wildlife, and transmission of the virus from livestock to wild animals or wild animals to livestock. Furthermore, the temporal and spatial dynamics of infection need to be conducted with the analysis of host animal distributions and contact opportunities, sero-surveys to estimate the level of infection and use of modern available techniques to track FMDV incursions into disease free areas.

\section{Conclusion}

We have detected the four FMDV serotypes O, A, SAT 1 and SAT 2, which are cocirculating in Tanzania. Sero-type A seems to occur in waves with periods (years) of apparent absence between epidemics. The presence of multiple serotypes and the complex epidemiology of FMD complicate the control of the disease through vaccination and establishment of FMDfree zones. Our findings also emphasize the importance of undertaking continuous surveillance to monitor the emergence and spread of FMDV strains. Therefore, further studies in both domestic and wild animals are required to investigate the genetic and antigenic characteristics of the circulating strains so that a rational control method for FMD in Tanzania and neighbouring countries can be recommended.

\section{Acknowledgements}

The authors wish to recognise the assistance provided by farmers and staff in Veterinary Investigation Centres for sample collection during FMD outbreaks. We appreciate the contribution of staff at the Central Veterinary Laboratory - Temeke during laboratory analysis of samples. We are grateful to the World Reference Laboratory (WRL), Pirbright, UK who provided ELISA kit for the study. The Ministry of Livestock Development and Fisheries, United Republic of Tanzania funded this work. The authors also acknowledge the Southern African Centre for Infectious Disease Surveillance for providing financial support (to CJK and RS) to present this paper at the first One Health conference in Africa, Johannesburg, South Africa, July 2011.

\section{References}

Di Nardo, A., Knowles, N.J. \& Paton, D.J., 2011, 'Combining livestock trade patterns with phylogenetics to help understand the spread of foot and mouth disease in sub-Saharan Africa, the Middle East and Southeast Asia', Revue Scientifique et Technique (OIE) 30, 63-85.

Ferris, N.P. \& Dawson, M., 1988, 'Routine application of enzyme-linked immunosorbent assay in comparison with complement fixation for the diagnosis of foot and mouth and swine vesicular diseases', Veterinary Microbiology 16, 201-209.

Hamblin, C., Armstrong, R.M. \& Hedger, R.S., 1984, 'A rapid enzyme linked immunosorbent assay for the detection of foot and mouth disease viruses in epithelial tissues', Veterinary Microbiology 9, 435-443.

Hedger, R.S., Forman, A.J. \& Woodford, M.H., 1973, 'FMDV in East African buffalo', Bulletin of Epizootic Diseases in Africa 21, 99-101.

Kivaria, F.M., 2003, 'Foot and mouth disease in Tanzania: an overview of its national status', Veterinary Quarterly 25, 72-78.

Knowles, N.J., Nazem Shirazi, M.H., Wadsworth, J., Swabey, K.G., Stirling, J.M., Statham, R.J. et al., 2009, 'Recent spread of new strain (A-Iran-05) of foot-andmouth disease virus type A in the Middle East', Transboundary and Emerging Tanzania: some problems associated with its control', Tanzania Veterinary Bulletin 5, 49-59.

MoWLD., 2003, 'Foot-and-mouth disease increases livestock keeper's poverty: let us stamp it out', Animal Health Newspaper 4, 1-12.

Paton, D.J., Sumption, K.J. \& Charleston, B., 2009, 'Options for control of foot-andmouth disease: knowledge, capability and policy', Philosophical Transactions of the Royal SocietyB 364, 2657-2667. http://dx.doi.org/10.1098/rstb.2009.0100

Perry, B.D. \& Rich, K.M., 2007, 'Poverty impacts of foot-and-mouth disease and the poverty reduction implications of its control', Veterinary Record 160, 238-241. http://dx.doi.org/10.1136/vr.160.7.238

Picado, A., Speybroeck, N., Kivaria, F., Mosha, R.M., Sumaye, R.D., Casal, J. \& Berkvens, D., 2010, 'Foot-and-Mouth Disease in Tanzania from 2001 to 2006 ' Transboundary and Emerging Diseases 58, 44-52. http://dx.doi.org/10.1111/ j.1865-1682.2010.01180.x

Rweyemamu, M.M. \& Loretu, K., 1972, 'Observations on foot-and-mouth disease in Tanzania', Bulletin of Epizootic Diseases of Africa 22, 101-104.

Rweyemamu, M.M. \& Loretu, K., 1973, 'Observations on the first epidemic of footand- mouth disease Type SAT 1 in Tanzania', Bulletin of Epizootic Diseases of Africa 21, 393-400.

Rweyemamu, M.M., 1984, 'Antigenic variation in foot-and-mouth disease: studies based on the virus neutralization reaction', Journal of Biological Standardization 12, 323-37. http://dx.doi.org/10.1016/S0092-1157(84)80013-X

Rweyemamu, M., Roeder, P., Mackay, D., Sumption, K., Brownlie, J., Leforban, Y. et al., 2008a, 'Epidemiological patterns of foot-and-mouth disease worldwide' Transboundary and Emerging Diseases 55, 57-72. http://dx.doi.org/10.1111/ j.1865-1682.2007.01013.x

Rweyemamu, M., Roeder, P., MacKay, D., Sumption, K., Brownlie, J. \& Leforban, Y., 2008b, 'Planning for the progressive control of foot-and-mouth disease worldwide', Transboundary and Emerging Diseases 55, 73-87. http://dx.doi. org/10.1111/j.1865-1682.2007.01016.x

Sahle, M., Dwarka, R.M., Venter, E.H. \& Vosloo, W., 2007, 'Comparison of SAT-1 footand mouth disease virus isolates obtained from East Africa between 1971 and 2000 with viruses from the rest of sub-Saharan Africa', Archives of Virology 152, 797-804. http://dx.doi.org/10.1007/s00705-006-0893-x

Swai, E.S., Mrosso, A. \& Masambu, J.I.G., 2009, 'Occurrence of foot-and-mouth disease serotypes in Tanzania: A retrospective study of tongue epithelial tissue samples' Tanzania Veterinary Journal 26, 7-12. http://dx.doi.org/10.4314/tvj.v26i1.49232

Vosloo, W., Bastos, A.D., Kirkbride, E., Esterhuysen, J.J., Janse van Rensburg D. Bengis., R.G. et al., 1996, 'Persistent infection of African buffalo (Syncerus caffer)
with SAT-type foot-and-mouth disease viruses: rate of fixation of mutations, antigenic change and interspecies transmission', Journal of General Virology 77 , 1457-1467. http://dx.doi.org/10.1099/0022-1317-77-7-1457

Vosloo, W., Bastos, A.D., Sangare, O., Hargreaves, S.K. \& Thomson, G.R., 2002, 'Review of the status and control of foot-and-mouth disease in sub-Saharan Africa', Revue Scientifique et Technique (OIE) 21, 437-44. 